\title{
ROTEIRO INSTRUCIONAL PARA A ELABORAÇÃo DE UM ESTUDO DE CASO CLÍNICO
}

\author{
Luzia Elaine Galdeano \\ Lídia Aparecida Rossi? \\ Márcia Maria Fontão Zago ${ }^{3}$
}

Galdeano LE, Rossi LA, Zago MMF. Roteiro instrucional para a elaboração de um estudo de caso clínico. Rev Latino-am Enfermagem 2003 maio-junho; 11(3):371-5.

Este trabalho teve como objetivo apresentar um roteiro instrucional para a elaboração de um estudo de caso clínico, com as finalidades de guiar o profissional de enfermagem, incentivar a reflexão acerca dos resultados encontrados e fornecer uma "seqüência" para a apresentação do estudo de caso e elaboração do relatório. O roteiro instrucional está constituído de: questões norteadoras; identificação; resumo dos problemas ou alterações identificadas; fundamentação teórica; alternativas ou propostas; ações implementadas ou recomendadas e discussão.

DESCRITORES: assistência ao paciente, ensino, relato de caso

\section{INSTRUCTIONAL SCRIPT FOR THE ELABORATION OF A CLINICAL CASE STUDY}

This study aimed to present an instructional script for the elaboration of a clinical case study, with a view to guiding nursing professionals, stimulating reflections about the results and providing a "sequence" for presenting the case study and elaborating the report. The instructional script consists of: guiding questions; identification; summary of the identified problems or changes; theoretical basis, alternatives or proposals; implemented or recommended actions and discussion.

DESCRIPTORS: patient care, teaching, case report

\section{guía INSTRUCTIVA PARA LA ELABORACIÓN DE UN ESTUdIO dE CASO}

Este trabajo tuvo como objetivo presentar una guía instructiva para la elaboración de un estudio de caso con la finalidad de guiar al profesional de enfermería, incentivar la reflexión acerca de los resultados encontrados y proporcionar una "secuencia" para la presentación del estudio de caso y la elaboración del informe. Dicha guía está constituida por: fundamentación teórica; alternativas o propuestas; acciones implementadas o recomendadas y discusión.

DESCRIPTORES: atención al paciente, enseñanza, informe de caso

\footnotetext{
${ }^{1}$ Professor Assistente da Faculdade de Enfermagem do Hospital Israelita Albert Einstein, e-mail: legaldeano@hotmail.com; ${ }^{2}$ Professor Associado, e-mail: rizzardo@eerp.usp.br; ${ }^{3}$ Professor Doutor, e-mail: mmfzago@eerp.usp.br. Escola de Enfermagem de Ribeirão Preto da Universidade de São Paulo, Centro Colaborador da OMS para o desenvolvimento da pesquisa em enfermagem
} 
INTRODUÇÃO

O estudo de caso é um dos mais antigos métodos utilizados no ensino de enfermagem e pode ser utilizado em educação continuada. Florence Nightingale já utilizava com seus alunos uma variação deste método de ensino, com a exigência de que os alunos levassem um caderno para registrar os casos excepcionalmente interessantes, sobre os quais seriam interrogados mais tarde para avaliar o que tinham aprendido ${ }^{(1)}$.

Com a evolução da profissão, o estudo, o ensino e a organização do cuidado de enfermagem tornaram-se focos de atenção do enfermeiro. Nesse contexto, surgem os estudos de caso, representando as primeiras tentativas de definição e sistematização da assistência de enfermagem. Os estudos de casos foram os precursores dos planos de cuidados, que, por sua vez, constituíramse nas primeiras expressões do Processo de Enfermagem, tão discutido atualmente ${ }^{(2)}$.

O surgimento do estudo de caso reflete o interesse da profissão em organizar o seu trabalho, baseando o estabelecimento de suas ações na análise da história do paciente.

A primeira descrição do método de estudo de caso na enfermagem foi publicada no Student's Handbook on Nursing Case Studies, em 1929, por Deborah Maclurg Jensen, com o objetivo de guiar o estudante na elaboração de estudos de caso, na Escola de Enfermagem da Universidade de Yale-EUA ${ }^{(2)}$.

No Brasil, a utilização de estudos de casos no ensino foi descrita em 1934. Nessa época, o estudo de caso era organizado em: história, sintomas subjetivos e objetivos, exames, diagnóstico social e médico, tratamentos médico e de enfermagem, complicações e $\operatorname{alta}^{(3)}$.

Nas publicações nacionais dessa época, encontramos estudos de casos compostos basicamente da descrição da história da doença, evolução da moléstia, tratamento médico e cuidado de enfermagem. Essas publicações refletem uma preocupação com o estabelecimento dos cuidados de enfermagem a serem executados com base no diagnóstico médico.

O estudo de caso pode ser definido como uma exploração de um sistema delimitado ou de um caso, obtido por meio de uma detalhada coleta de dados, envolvendo múltiplas fontes de informações ${ }^{(4)}$. É um estudo aprofundado de uma unidade, grupo ou indivíduo, em sua complexidade e em seu dinamismo próprio, fornecendo informações relevantes para a tomada de decisão ${ }^{(5)}$.

Observamos que os termos exploração detalhada e estudo profundo aparecem nas definições de estudo de caso; o objetivo é ressaltar o rigor da coleta de dados de um ou de vários casos, fornecendo um relatório organizado das variáveis, visando facilitar a análise dos dados e a tomada de decisões.

Podemos classificar os estudos de casos em dois tipos: os estudos de casos formais utilizados por pesquisadores para descrever, analisar e entender determinados fenômenos; e os estudos de casos informais apropriados para a prática clínica, quando se deseja analisar ou descrever uma situação particular, identificar problemas em determinados campos, observar mudanças e explorar as causas ${ }^{(6)}$.

Chamamos de estudos de casos formais uma diversidade de pesquisas que têm por objetivos coletar e registrar dados de um ou vários casos, a fim de organizar um relatório ordenado e crítico, e avaliar profundamente os dados coletados, com o objetivo de tomar decisões e propor ações transformadoras ${ }^{(6)}$.

O estudo de caso é um método muito utilizado em pesquisas qualitativas, desenvolvendo-se em uma situação natural, rica em dados descritivos e que focaliza a realidade de uma forma complexa e contextualizada. Esse método também é utilizado em estudos quantitativos, como, por exemplo, estudos de casos clínicos, de direito, de serviço social, entre outros ${ }^{(5)}$. É importante compreender que, se o interesse do pesquisador é desenvolver um estudo de caso centrado na manipulação e mensuração de variáveis fisiológicas e, a partir daí, instituir algum tipo de intervenção, o estudo de caso será quantitativo ${ }^{(7)}$. Nesse tipo de estudo, o pesquisador é um observador passivo, reunindo informações sobre os comportamentos, sintomas e características da pessoa, à medida que eles, naturalmente, são verificados ${ }^{(8)}$.

Os estudos de casos clínicos, também chamados de estudos de casos informais, são os estudos aplicados na assistência direta de enfermagem, com o objetivo de realizar um estudo profundo dos problemas e necessidades do paciente, família e comunidade, proporcionando subsídios para enfermeira estudar a melhor estratégia para solucionar ou reverter os problemas identificados. Para aplicar o estudo de caso, a enfermeira deve ter conhecimento não somente das técnicas de enfermagem, mas também sobre a fisiopatologia das doenças, sinais e 
sintomas e fatores socioeconômicos envolvidos no processo saúde-doença ${ }^{(1)}$.

O estudo de caso clínico fundamenta as ações de enfermagem; proporciona uma assistência individual e personalizada, na qual o paciente é visto como um ser único e não como um conjunto de sinais e sintomas; proporciona um elo entre as diversas áreas que atuam de forma intervencionista nos problemas do paciente; proporciona uma familiarização da enfermeira com a literatura científica, utilizada para embasar suas decisões; contribui na formação de um corpo concreto de conhecimento de enfermagem, pois os registros e arquivos dos estudos de casos podem ser utilizados como referência futura e contribui para melhorar o desempenho da equipe de enfermagem ${ }^{(1)}$.

O estudo de um caso permite que o profissional observe, entenda, analise e descreva uma determinada situação real, adquirindo conhecimento e experiência que podem ser úteis na tomada de decisão frente a outras situações. É um método de investigação no qual o profissional tem um grande envolvimento e que inclui, como etapas, a coleta de informações, um processo de pensamento, constituído por análise dos dados e determinação de soluções, e um processo de julgamento ou avaliação ${ }^{(9)}$. A expectativa é que o profissional adquira conhecimento e experiência para tomar decisões e resolver os problemas identificados no estudo de caso ${ }^{(10)}$.

O estudo de caso clínico teve maior destaque no ensino, com objetivo de favorecer a aprendizagem de enfermagem no campo de estágio ${ }^{(10-12)}$. Os alunos de enfermagem são orientados a estudar, com profundidade, os problemas fisiológicos, sociais e espirituais do paciente para o qual prestam cuidados durante as atividades práticas de determinadas disciplinas.

O valor prático do estudo e análise do caso é prover uma oportunidade de examinar uma situação de vida real. O estudante pode determinar a causa específica do problema, discutir como preveni-lo e sugerir várias soluções. Esse método viabiliza uma maior correlação da teoria com a prática, favorece a assimilação e aplicação prática do ensino teórico pelos alunos, além de proporcionar um aprofundamento da fisiopatologia, e favorecer a definição de ações ${ }^{(13)}$.

O estudo de caso é uma estratégia de ensino humanista e problematizadora que contribui, de forma muito positiva, para a formação do aluno, pois aumenta o conhecimento teórico-prático referente à assistência, estimula a autonomia do aluno na tomada de decisões, na solução de problemas e no conhecimento referente à metodologia científica ${ }^{(11)}$.

Apesar da sua grande aplicabilidade, muitos ainda acreditam que a elaboração do estudo de caso consiste, basicamente, em descrever a história do paciente, os sinais e sintomas (objetivos e subjetivos), os exames, o diagnóstico médico e o tratamento instituído. No entanto, devemos ressaltar que a coleta de informações constitui, apenas, uma das fases da elaboração do estudo de caso, seguida de um processo de pensamento, que corresponde à fase de categorização e análise dos dados, uma fase de determinação de estratégias para solucionar ou amenizar os problemas identificados e uma fase de avaliação ou julgamento. Devido a esse fato e considerando as contribuições dos estudos de casos clínicos (informais) na assistência e no ensino de enfermagem, elaboramos um roteiro instrucional com o objetivo de guiar o profissional ou aluno na elaboração de um estudo de caso informal ou estudo de caso clínico.

\section{ROTEIRO INSTRUCIONAL - ESTUDO DE CASO INFORMAL}

Para iniciar um estudo de caso, seja a área qual for, é necessário construir um manual de orientação, com o objetivo de instruir o profissional ou aluno quanto aos aspectos mais importantes relacionados ao referido caso, incentivar a reflexão acerca dos resultados encontrados e fornecer uma "seqüência" para a apresentação do estudo de caso e elaboração do relatório. No nosso entender, trata-se de roteiro para a elaboração do estudo de caso, com a finalidade de guiar o profissional e assegurar que não sejam esquecidos ou omitidos dados considerados essenciais $^{(9)}$.

O roteiro instrucional elaborado está constituído de: (1) questões norteadoras; (2) identificação (do local ou pessoa em estudo); (3) resumo dos problemas ou alterações identificados; (4) fundamentação teórica; (5) alternativas ou propostas; (6) ações implementadas ou recomendas e (7) discussão.

\section{(1) Questões norteadoras}

Como o próprio nome diz, as questões norteadoras irão nortear o estudo de caso. Em geral, usamos as seguintes questões: quê? quem? como? por quê? e qual? Exemplificando: 
- quemé a pessoa envolvida no caso ou qual é o lugar que está sendo estudado?;

- o que aconteceu? ou qualé o problema?;

- como aconteceu? é importante seguir uma ordem cronológica;

- por que aconteceu?;

- quais as alternativas para solucionar ou amenizar os problemas identificados?;

- que soluções ou alternativas estão sendo propostas?

É importante que, ao final do estudo de caso, todas essas questões sejam esclarecidas.

\section{(2) Identificação}

Essa fase é muito importante para o desenvolvimento do estudo de caso, consiste na fase de coleta de informações, que deve ser realizada, utilizandose várias fontes de informação (entrevista, observação, exame físico, prontuário do paciente, familiares, etc) ${ }^{(14)}$. O objetivo é investigar, com profundidade, a pessoa ou local em estudo.

Nessa etapa, é importante que se construa um instrumento de coleta de dados, para servir de guia de orientação para o profissional, permitindo o direcionamento e o registro das observações realizadas e assegurando que não sejam omitidos dados considerados essenciais para investigar e explorar o que se deseja. A estrutura do instrumento de coleta deve retratar o referencial teórico adotado, a dinâmica do serviço, o padrão de organização da assistência e a especificidade da clientela assistida ${ }^{(15)}$.

A decisão sobre desenvolver um estudo de caso normalmente ocorre mediante a observação de um problema que merece um estudo mais aprofundado ou necessita dele, podendo ser, por exemplo, o padrão respiratório ineficaz em pacientes cardíacos, ou ainda, integridade da pele prejudicada em pacientes acamados. No nosso exemplo, o padrão respiratório ineficaz ou a integridade da pele prejudicada seria nosso "foco de atenção". No entanto, ao desenvolver o estudo de caso, não podemos restringir a investigação ao foco de atenção, já que procuramos entender não somente o que aconteceu ou qual o problema, mas também como, por que e qual a sua relação com as outras alterações identificadas. Ressaltamos que o paciente deve ser avaliado de maneira integral e individualizada, considerando seus aspectos biopsicossociais.

(3) Resumo dos problemas ou alterações identificados

Essa fase consiste em analisar e categorizar os dados para o levantamento de problemas de enfermagem.
Corresponde ao momento em que os dados são agrupados para caracterizar e definir determinado problema.

No relatório, deve constar um resumo das alterações identificadas nos diversos sistemas (respiratório, cardiovascular, etc.) ou necessidades básicas (oxigenação, circulação, termorregulação, etc.), dependendo do referencial teórico adotado.

Para exemplificar, apresentamos, a seguir, um modelo de categorização dos problemas, considerando no exemplo, dados de um paciente que será submetido a uma revascularização do miocárdio, utilizando-se, como referencial teórico, o modelo conceitual de Wanda Horta, que se baseia na teoria das necessidades básicas:

a) necessidade de oxigenação - dispnéia, ortopnéia, dispnéia paroxística noturna e murmúrios vesiculares diminuídos em ápice pulmonar esquerdo;

b) necessidade de circulação - paciente com diagnóstico médico de doença arterial corionariana e hipertensão será submetido a uma revascularização do miocárdio, ao exame $P A=200 \times 100 \mathrm{mmHg}$ e pulso apical arrítmico, apresenta edema (+++/4+) maleolar bilateral e trajetos varicosos bilaterais em membros inferiores;

c) necessidade de integridade física - paciente refere não seguir as orientações médicas e não fazer uso dos medicamentos prescritos, refere ser tabagista há 30 anos (média de dois maços por dia);

d) necessidade de segurança - paciente refere estar extremamente ansioso em relação à cirurgia e ter muito medo de morrer.

\section{(4) Fundamentação teórica}

Nesse momento, é fundamental o aprofundamento da fisiopatologia, procurando buscar informações que justifiquem as alterações ou problemas identificados. Dessa forma, procuramos, embasados na literatura, responder como? e por quê? à presente situação.

Baseando-se nos dados apresentados no exemplo anterior, podemos observar que o paciente apresenta, entre outros problemas: dispnéia, ortopnéia e dispnéia paroxística noturna. Logo, esta fase consiste em identificar como? e por quê? essas alterações estão presentes e qual a relação com o diagnóstico do paciente. Desse modo, cada alteração encontrada deve ser fundamentada na literatura.

Apresentamos, a seguir, um exemplo da fase de fundamentação teórica, considerando os problemas discutidos acima - dispnéia/ortopnéia/dispnéia paroxística noturna e doença arterial coronariana. 
"A dispnéia de origem cardiovascular é resultante da insuficiência cardíaca, uma vez que reflete a incapacidade do coração em produzir um débito cardíaco suficiente para satisfazer às necessidades teciduais de oxigênio"(16). "A ortopnéia e a dispnéia paroxística noturna também são manifestações da insuficiência cardíaca, e ocorrem em pacientes com edema periférico cujo líquido intersticial é reabsorvido para a circulação central, quando o paciente se encontra em decúbito dorsal, levando-o a uma sensação de falta de $\operatorname{ar}^{\text {"(17). }}$.

\section{(5) Alternativas ou propostas}

A quinta etapa corresponde em buscar na literatura as estratégias ou alternativas existentes para a resolução dos problemas identificados.

É importante entender e descrever essas alternativas de forma a identificar a melhor proposta para o problema identificado e ajudar na tomada de decisões.

\section{(6) Ações implementadas ou recomendas}

Essa etapa consiste em descrever a alternativa escolhida para reverter ou amenizar os problemas identificados, justificando o porquê da escolha.

Caso a alternativa ou proposta escolhida seja colocada em prática é importante acompanhar a evolução, registrando as mudanças.

\section{REFERÊNCIAS BIBLIOGRÁFICAS}

1. Heidgerken LE. Enseñanza de la Enfermeria. $2^{\text {a }}$ ed. México: Interamericana; 1963.

2. Henderson V. On nursing care plane and their history. Nurs Outlook 1973 June; 21(6):378-9.

3. Vidal ZC. "O caso de estudo". Annaes de Enfermagem $1934 ; 5(5): 28-30$.

4. Creswell JW. Qualitative inquiry and research design choosing among five traditions. London: Sage; 1998.

5. Lüdke M, André MEDA. Pesquisa em educação abordagens qualitativas. São Paulo: EPU; 1986.

6. Moon SM, Trepper TS. Case study research. In: Sprenkle $\mathrm{DH}$, Moon SM. Research methods in familiy therapy. New York: Guilford Press; 1996. p.393-410.

7. Garcia TR. Cuidando de adolescentes grávidas. [tese]. Ribeirão Preto: Escola de Enfermagem de Ribeirão Preto/ USP; 1996.

8. Polit D, Hungler BP. Fundamentos de pesquisa em enfermagem. Porto Alegre: Artes Médicas Sul; 1995.

9. Naumes W, Naumes MJ. The art and craft of case writing. Philadefhia: Sage; 1999.

10. Tarcinale M. A the case study as a vicarious learning technique. J Nurs Education 1987 October; 26(8):340-1.

\section{(7) Discussão}

Corresponde apresentação e discussão do estudo de caso com outros profissionais. Essa etapa tem por objetivo envolver os profissionais no caso estudado, incentivar um processo de pensamento e julgamento, levantando discussões que irão determinar outras propostas e troca de experiências, resultando em um processo de decisão e avaliação.

É fundamental a elaboração de um relatório, descrevendo todas as etapas anteriores.

\section{CONSIDERAÇÕES FINAIS}

O estudo de caso é um método amplo que permite ser aplicado a uma grande variedade de problemas e contribui, de forma consistente, para o desenvolvimento de um corpo de conhecimento próprio em enfermagem.

Em todas as áreas, os estudos de casos são desenvolvidos para proporcionar um maior conhecimento e envolvimento do profissional, aluno ou pesquisador, com uma situação (real) observada. O objetivo é descrever, entender, avaliar e explorar essa situação, e, a partir daí, determinar os fatores causais e estabelecer ações.

11. Bocchi SCM. Modelo operacional do estudo de caso como estratégia de ensino na disciplina de enfermagem médicocirúrgica - avaliação dos alunos. Rev Latino-am Enfermagem 1996 dezembro; 4(3):99-116.

12. Johnson J, Purvis MSN. Case studies - an alternative learning/teaching method in nursing. J Nurs Education 1987 March; 26(3):118-20.

13. Martins RC, Fonseca AS. O estudo de caso como estratégia de ensino no curso de graduação em enfermagem da Fundação Municipal de Ensino Superior de Marília. Rev Bras Enfermagem 1992 abril/set; 45(2/3):231-8.

14. Yin RK. Case study research - design and methods. $2^{\mathrm{a}}$ ed. Philadefhia: Sage; 1994.

15. Dalri MCB. Perfil diagnóstico de pacientes queimados segundo modelo conceitual de Horta e a Taxonomia I da NANDA. [dissertação]. Ribeirão Preto: Escola de Enfermagem de Ribeirão Preto/USP; 1993.

16. Andreoli TE, Bennett JC, Carpenter CCJ, Plum Jr F, Smith LH. Doenças cardiovasculares. In: Andreoli TE, Bennett JC, Carpenter CCJ, Plum Jr F, Smith LH. CECIL: Medicina interna básica. Rio de Janeiro: Guanabara Koogan; 1994. p.2-212. 17. Braunwald EB. Exame clínico. In: Goldman L, Braunwald EB. Cardiologia na clínica geral. Rio de Janeiro: Guanabara Koogan; 2000. p.26-42. 